\title{
Conceptual Design of Low-Boom Aircraft with Flight Trim Requirement
}

\author{
Irian Ordaz* and Karl A. Geiselhart ${ }^{\dagger}$ \\ NASA Langley Research Center, Hampton, VA 23681 \\ and James W. Fenbert ${ }^{\ddagger}$ \\ Analytical Mechanics Associates, Inc., Hampton, VA 23681
}

\begin{abstract}
A new low-boom target generation approach is presented which allows the introduction of a trim requirement during the early conceptual design of supersonic aircraft. The formulation provides an approximation of the center of pressure for a presumed aircraft configuration with a reversed equivalent area matching a low-boom equivalent area target. The center of pressure is approximated from a surrogate lift distribution that is based on the lift component of the classical equivalent area. The assumptions of the formulation are verified to be sufficiently accurate for a supersonic aircraft of high fineness ratio through three case studies. The first two quantify and verify the accuracy and the sensitivity of the surrogate center of pressure corresponding to shape deformation of lifting components. The third verification case shows the capability of the approach to achieve a trim state while maintaining the low-boom characteristics of a previously untrimmed configuration. Finally, the new low-boom target generation approach is demonstrated through the early conceptual design of a demonstrator concept that is low-boom feasible, trimmed, and stable in cruise.
\end{abstract}

\section{Nomenclature}

Acronyms

CFD computational fluid dynamics

GA genetic algorithm

Symbols

$A_{\mathrm{e}} \quad$ equivalent area

$A_{\mathrm{e}}^{\text {lift }} \quad$ lift component of classical equivalent area

$A_{\mathrm{e}}^{\mathrm{Mach}} \quad$ classical equivalent area

$A_{\mathrm{e}}^{\mathrm{rev}} \quad$ reversed equivalent area

$\alpha \quad$ angle of attack

$\mathrm{cg} \quad$ location of center of gravity

$c g_{x} \quad$ axial location of center of gravity

$C_{L} \quad$ coefficient of lift

cp location of center of pressure

$c p_{x} \quad$ axial location of center of pressure

$\Delta A_{\mathrm{e}} \quad$ difference between the reversed and classical equivalent area

\footnotetext{
*Aerospace Engineer, Aeronautics Systems Analysis Branch, Mail Stop 442.

${ }^{\dagger}$ Aerospace Engineer, Aeronautics Systems Analysis Branch, Mail Stop 442.

¥Aerospace Engineer, Aeronautics Systems Analysis Branch, Mail Stop 442.
} 


$\begin{array}{ll}\Delta p & \text { ground pressure sign } \\ L & \text { lift force } \\ \mu & \text { Mach angle } \\ \phi & \text { azimuthal direction } \\ \rho_{\infty} & \text { freestream density } \\ U_{\infty} & \text { freestream velocity }\end{array}$

\section{Introduction and Motivation}

7 He design and optimization of a low-boom supersonic aircraft requires accurate modeling of sonic 1 boom phenomena and exploration of a complex design space, often with contradicting environmental and performance objectives. The shape optimization process used to mitigate the sonic boom of an aircraft can lead to compromises in flight performance, and in some cases low-boom configurations that are not capable of achieving a trimmed state.

Mitigation of sonic boom can be performed through a direct optimization approach, where a numerical optimizer is used to minimize maximum overpressure, initial shock pressure rise, or perceived loudness of a ground signature. Additional constraints such as the location of the center of pressure $(c p)$ can be included to account for the trim requirement during cruise. However, such approaches only provide new information regarding the feasibility of a new design through expensive computational simulation. It is impossible to know a priori if the design effort can be successful.

The inverse design approach attempts to drive the design toward an ideal definition of a sonic boom metric referred to as a target. Low-boom optimization ${ }^{1-3}$ is more often performed using this approach since there is a natural decoupling in the sonic boom analysis requirements as shown in Fig. 1. A pressure distribution calculated sufficiently far away from the aircraft (typically 3-5 body lengths ${ }^{4}$ ) can be propagated to the ground using only an atmospheric propagation code. Candidate pressure or equivalent area distributions $\left(A_{\mathrm{e}}\right)$ for low-boom targets are defined at this off-body location because sonic boom analysis based strictly on atmospheric propagation is less computationally expensive. This allows rapid exploration of the target

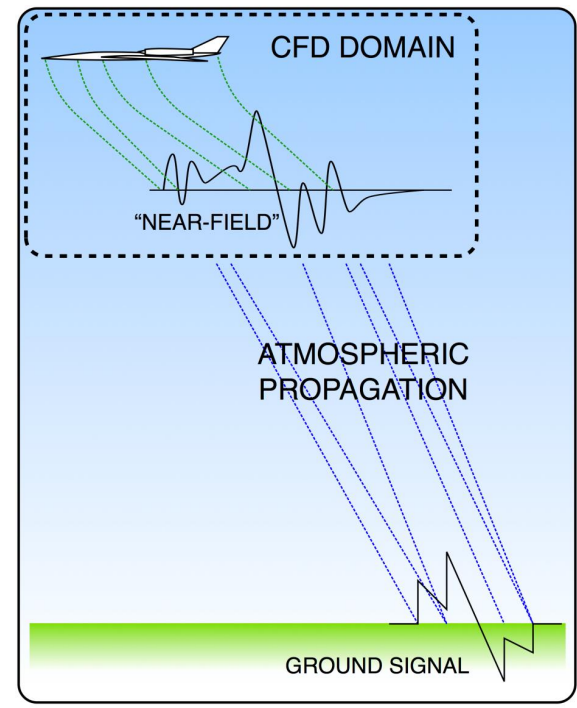

Figure 1. Overview of sonic boom analysis methodology (from Ref. 1). design space and can provide feasibility information early in the design process.

Traditionally, these low-boom targets minimized sonic boom metrics but were unable to capture other important requirements such as trim at cruise. ${ }^{5}$ In addition, shaping a configuration to match a lowboom target often requires aft lift tailoring ${ }^{6}$ which can result in an aft shift of the axial center of pressure $\left(c p_{x}\right)$, potentially leading to an untrimmed aircraft. Recovering trim characteristics after the aircraft has been shaped for low-boom is not always possible, and at best, results in a compromised design. The redistribution of lift fore of the axial center of gravity $\left(c g_{x}\right)$ through the use of canards, strakes, and other lifting devices can also lead to structurally unacceptable configurations due to the volume trade necessary to maintain low-boom.

This paper presents a new approach for generating low-boom targets which are capable of driving the design toward a trimmed state. The formulation uses a surrogate lift distribution to predict the location of $c p_{x}$ corresponding to a presumed aircraft geometry with a reversed equivalent area ${ }^{7}\left(A_{\mathrm{e}}^{\mathrm{rev}}\right)$ that matches the target $A_{\mathrm{e}}$. A description of the sonic boom analysis including CFD and atmospheric propagation is provided in Section II. The formulation and assumptions used to approximate $c p_{x}$, along with numerical verification is presented in Section III. The initial conceptual design of a trimmed and stable low-boom demonstrator including mass properties, mission performance, trim-feasible target generation, and preliminary shaping is presented in Section IV. Finally, a summary of the work is presented in Section V. 


\section{Description of the Sonic Boom Analysis}

This section describes the CFD analysis and propagation tool used during the conceptual design process of a low-boom demonstrator (Section IV). The analysis codes described are integrated into a ModelCenter ${ }^{8}$ model that allows early conceptual design and layout of a configuration using the mixedfidelity $A_{\mathrm{e}}$ approach described in Ref. 9.

\section{A. CFD Analysis for Sonic Boom}

The CFD analysis is conducted with Cart3D, ${ }^{10,11}$ an inviscid CFD analysis package that is geared toward conceptual and preliminary aerodynamic design. The package allows for quick and automated Cartesianbased volume mesh generation and CFD solutions for complex geometries. The volume mesh used for sonic boom analysis is rotated by the Mach angle to align the shocks with the computational grid and relies on an automated approach that places refinement boxes in the region of interest. This region typically extends 3-5 body lengths away from the aircraft, where three-dimensional effects are fully resolved before propagation with an atmospheric propagation code. The automated Cart3D off-body analysis procedure, with a stretched and rotated grid, is verified in Ref. 6 through numerical comparison to USM3D. ${ }^{12}$

\section{B. Sonic Boom Analysis}

The off-body pressure distribution calculated with Cart3D is propagated with the sBOOM ${ }^{13}$ atmospheric propagation code to obtain the ground signatures and perceived loudness. By solving the augmented Burgers equation, sBOOM takes into account atmospheric losses due to nonlinearity, molecular relaxation, and thermo-viscous absorption.

sBOOM is also used to calculate the $A_{\mathrm{e}}^{\text {rev }}$ which is part of the mixed-fidelity approach used in the design process of the low-boom demonstrator concept (Section IV). The $A_{\mathrm{e}}^{\text {rev }}$ is calculated from an off-body pressure distribution with fully resolved three-dimensional aerodynamic effects. The pressure distribution is propagated backward in time with sBOOM to a location near the configuration, and converted into an equivalent area. The $A_{\mathrm{e}}^{\mathrm{rev}}$ is a better representation of the aircraft as a body-ofrevolution than the classical equivalent area $\left(A_{\mathrm{e}}^{\mathrm{Mach}}\right)$. It has been verified that in practice, the ground signature propagated from $A_{\mathrm{e}}^{\mathrm{rev}}$ is the same as the ground signature propagated from the off-body pressure distribution: the two signatures have the same shape and same level of perceived loudness. ${ }^{7}$ The $A_{\mathrm{e}}^{\text {rev }}$ is useful during early conceptual design because it provides a mapping of the variation between the sonic boom characteristics of a configuration and a low-boom target.

\section{Generation of Trim-Feasible Low-Boom Targets}

\section{A. Calculation of a Surrogate Center of Pressure}

The trim-feasible target formulation is based on the mixed-fidelity $A_{\mathrm{e}}$ design approach. ${ }^{9}$ The design methodology shows that the sensitivity of $A_{\mathrm{e}}^{\text {rev }}$ to design changes can be approximated by the sensitivity of $A_{\mathrm{e}}^{\mathrm{Mach}}$, which is based on a surface pressure distribution (see Eq. 1). Here, the $\Delta A_{\mathrm{e}}$ of a configuration is defined as the difference between $A_{\mathrm{e}}^{\mathrm{rev}}$ and $A_{\mathrm{e}}^{\mathrm{Mach}}$. According to the mixed-fidelity approach, the difference in $\Delta A_{\mathrm{e}}$ between the baseline and design configurations as a result of small variations in the lift distribution and total lift is also small.

The mixed-fidelity equivalent area $\left(A_{\mathrm{e}}^{\text {mixed }}\right)$ in Eq. 1, which is an approximation of $A_{\mathrm{e}}^{\text {rev }}$ for the next design iteration, is set equal to the target $A_{\mathrm{e}}$. The $A_{\mathrm{e}}^{\mathrm{Mach}}$ terms in Eq. 1 can be decomposed into body (volume) and lift components, ${ }^{14}$ and the difference in volume $A_{\mathrm{e}}$ between the baseline and design configurations is assumed to be small. This is a reasonable assumption if lift tailoring is used to match the target $A_{\mathrm{e}}$. Once the volume components of $A_{\mathrm{e}}^{\mathrm{Mach}}$ for the baseline and design configurations are eliminated, the surrogate $A_{\mathrm{e}}$ due to lift for the design $\left(A_{\mathrm{e}, \mathrm{design}}^{\mathrm{lift}}\right)$ is calculated using Eq. 2.

$$
\begin{aligned}
& A_{\mathrm{e}}^{\text {mixed }}=A_{\mathrm{e}, \text { baseline }}^{\text {rev }}-A_{\mathrm{e}, \text { baseline }}^{\mathrm{Mach}}+A_{\mathrm{e}, \text { design }}^{\mathrm{Mach}} \\
& A_{\mathrm{e}, \text { design }}^{\text {lift }}=A_{\mathrm{e}, \text { target }}-A_{\mathrm{e}, \text { baseline }}^{\text {rev }}+A_{\mathrm{e} \text {, baseline }}^{\text {lift }}
\end{aligned}
$$

The surrogate $A_{\mathrm{e}}$ due to lift is used to approximate the lift distribution along the aircraft axial direction. The relation between $A_{\mathrm{e}}$ due to lift and the aircraft lift distribution shown in Eq. 3 is given by Walkden ${ }^{14}$ for an axial station $(x)$ and azimuthal direction $(\phi)$. Note that in this equation, the lift distribution is calculated by integrating the surface pressure up to an equivalent $x$-location $\left(x_{\mathrm{e}}\right)$ where the Mach plane intersects the configuration. An assumption is made that the contribution of drag force 
to pitching moment is relatively small in comparison to the lifting force. This is a reasonable assumption for low-boom supersonic aircraft which typically have a high fineness ratio. Therefore, the contribution to pitching moment can be approximated using only the lift distribution $\left(L\left(x_{\mathrm{e}}, \phi\right)\right)$ in Eq. 3 . The lift contribution to pitching moment of the interval defined by Mach planes located at $x_{\mathrm{e}, i}$ and $x_{\mathrm{e}, i+1}$ is calculated through the difference in $A_{\mathrm{e}}^{\text {lift }}$ at these Mach plane locations as shown in Fig. 2, and the discrete equation is given in Eq. 4 for $\phi$ equal to zero $(x-z$ plane at $y=0)$.

$$
\begin{gathered}
A_{\mathrm{e}}^{\mathrm{lift}}\left(x_{\mathrm{e}}, \phi\right)=\frac{\beta}{\rho_{\infty} U_{\infty}^{2}} \int_{0}^{x_{\mathrm{e}}} L\left(x_{\mathrm{e}}, \phi\right) \\
L_{i}=\frac{\rho_{\infty} U_{\infty}^{2}}{\beta}\left(A_{\mathrm{e}, i}^{\mathrm{lift}}-A_{\mathrm{e}, i-1}^{\mathrm{lift}}\right) \\
c p_{x}=\frac{\sum_{i=1}^{n} L_{i} x_{i}}{\sum_{i=1}^{n} L_{i}}
\end{gathered}
$$

The point highlighted in red in Fig. 2 represents the centroid of the $i$-th Mach plane section on the baseline configuration. The $x$-location of this section centroid is used as the pitching moment arm for the lift contribution at the $i$-th section. The variation of the centroid location of each Mach plane section after a design iteration is assumed to be small. This assumption is a reasonable if the configuration components are not re-arranged and any changes in angle-of-attack $(\alpha)$ are small. Otherwise, the Mach plane section centroids and CFD solution need to be re-calculated for the new baseline configuration with re-arranged components. The predicted location of $c p_{x}$ can be calculated using the lift distribution and section centroids $\left(x_{i}\right)$ as shown in Eq. 5, where $n$ is the total number of sections.

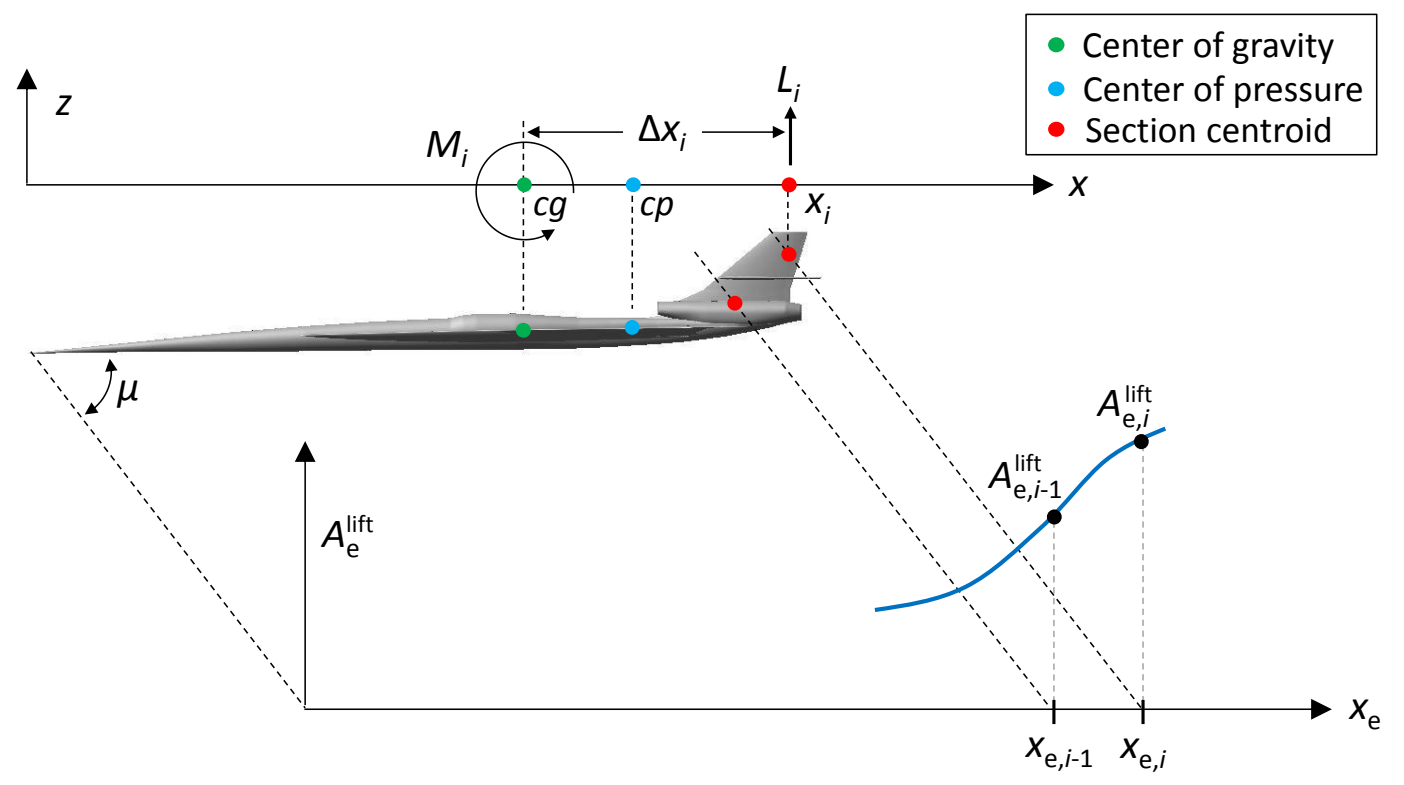

Figure 2. Calculation of moment about the center of gravity using $A_{\mathrm{e}}$ due to lift.

\section{B. Formulation of the Optimization Problem}

The existing in-house target generation process is integrated in ModelCenter and uses a Bézier spline defined by an arbitrary number of control points to represent the target $A_{\mathrm{e}}$. A total of 8 control points are used for the trim-feasible target optimization that is presented. The location of the first and last control point is maintained fixed, but the entire curve is allowed to scale based on the end value of the $A_{\mathrm{e}}$ which is a function of aircraft flight conditions. A genetic algorithm (GA) is used due to its robustness when handling the large number of local minima present in the perceived loudness objective function. The GA is used to minimize the perceived loudness level by varying the coordinate location of the spline control points which define the target $A_{\mathrm{e}}$. Each objective function evaluation requires the conversion of the target $A_{\mathrm{e}}$ to a pressure distribution which is then propagated using sBOOM to obtain a ground signature and a perceived loudness level.

The integration of a trim requirement into the low-boom target generation process is achieved through the use of the previously described formulation which provides a surrogate $c p_{x}$ location. The new formulation for the generation of trim-feasible low-boom targets aims to minimize both, the perceived loudness 
and the surrogate metric for $c p_{x}$. A non-dominated sorting genetic algorithm ${ }^{15}$ (NSGA-II) distributed with ModelCenter is used to optimize these objectives and create a Pareto front which allows the designer to perform trades between translation of $c p_{x}$ and loudness level for the new design iteration. A diagram of the optimization process and flow of parameters is shown in Fig. 3.

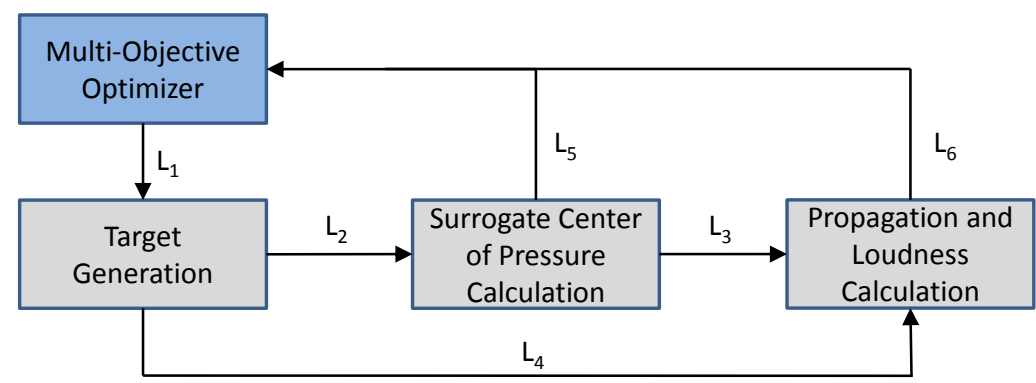

Linked Parameters:

$\mathrm{L}_{1}:$ Spline control points for target $A_{\mathrm{e}}$

$\mathrm{L}_{2}$ : Target $A_{\mathrm{e}}$

$\mathrm{L}_{3}$ : Propagation altitude based on end value of surrogate $A_{\mathrm{e}}$ due to lift

$\mathrm{L}_{4}$ : Target $A_{\mathrm{e}}$

$\mathrm{L}_{5}$ : Change in surrogate $c p_{x}$

$\mathrm{L}_{6}$ : Perceived loudness

Figure 3. Optimization process.

Optimization constraints are placed on the location of the spline control points and on the upper limit of the end value of the target $A_{\mathrm{e}}$. If the cruise weight and Mach number are assumed constant across design iterations, then constraining the end value of the target $A_{\mathrm{e}}$ to that of the baseline $A_{\mathrm{e}}^{\text {rev implicitly }}$ sets the target cruise altitude to equal that of the baseline configuration. Constraining the cruise altitude to that of the baseline provides the most accurate sensitivity of surrogate $c p_{x}$ to shape changes. This is because the $\Delta A_{\mathrm{e}}$ of the target is expected to remain similar to that of the baseline configuration. However, constraining the cruise altitude also limits the design space and can produce a more heavily compromised target $A_{\mathrm{e}}$.

The process of increasing the pitch up moment to shift $c p_{x}$ forward and match the $c g_{x}$ requires adding positive lift fore of the $c g_{x}$ and removing lift aft of the $c g_{x}$. If the change in target $A_{\mathrm{e}}$ is assumed to be mainly a result of changes in $A_{\mathrm{e}}^{\text {lift }}$ as shown by the mixed-fidelity approach, then this change in lift distribution manifests itself as (i) an increase in target $A_{\mathrm{e}}$ fore of the $x_{e}$ location of $c g_{x}$, (ii) a decrease or flattening of the target $A_{\mathrm{e}}$ aft of the $x_{e}$ location of $c g_{x}$, or (iii) a combination of the two scenarios. If the end value of the target $A_{\mathrm{e}}$ is fixed then this can drive the target $A_{\mathrm{e}}$ toward a design space region that is less amenable to low-boom.

The solution for this over-constrained problem is to relax the end value constraint of the target $A_{\mathrm{e}}$. Allowing the end $A_{\mathrm{e}}$ value to change implicitly allows the cruise altitude to vary for new design iterations. This requires either a re-design of the configuration, or a change in $\alpha$ to match the new required $C_{L}$. Varying $\alpha$ is the simplest way to achieve the $C_{L}$ needed for the new cruise altitude. However, this introduces a challenge because changes in $\alpha$ typically violate the stated assumption that $\Delta A_{\mathrm{e}}$ for the new design iteration can be approximated using the $\Delta A_{\mathrm{e}}$ of the baseline configuration. Sensitivity studies with respect to $\alpha$ show that the $c p_{x}$ that is calculated using strictly the lift contribution from $A_{\mathrm{e}}^{\text {lift }}$ can become unreliable for predicting the expected change in actual $c p_{x}$. Cases where $\alpha$ has to be adjusted to match the new design $C_{L}$ need to be solved iteratively by allowing only small changes in design cruise altitude (or end value of target $A_{\mathrm{e}}$ ), and calculating a new CFD solution for the baseline configuration at the new design cruise altitude and $C_{L}$. This provides at each iteration of the target optimization an updated $\Delta A_{\mathrm{e}}$ which makes prediction of the change in $c p_{x}$ more accurate.

The cruise altitude corresponding to a low-boom target $A_{\mathrm{e}}$ is calculated using the end value of the $A_{\mathrm{e}}$ assuming constant Mach number and aircraft weight. Recall that in this context, the target $A_{\mathrm{e}}$ can be thought of as the $A_{\mathrm{e}}^{\text {rev }}$ of a presumed design configuration. The end value of the surrogate $A_{\mathrm{e}}^{\text {lift }}$ given by Eq. 2 is scaled based on the ratio of the end values of $A_{\mathrm{e}}^{\text {lift }}$ and $A_{\mathrm{e}}^{\mathrm{rev}}$ for the baseline configuration. This assumption is possible because in practice, the variation of this ratio as a result of local shape deformation and changes in $\alpha$ is small. In addition, the scaling of the end value of the surrogate $A_{\mathrm{e}}^{\text {lift }}$ is mathematically consistent because a constant scaling of the surrogate lift distribution does not affect the calculation of the surrogate $c p_{x}$. 


\section{Verification of Center of Pressure Sensitivities}

\section{Sensitivities Based on Shaping for a Wing-Body Configuration}

Three studies are conducted to verify the sensitivity in the translation of $c p_{x}$ due to shape changes. The first case uses a simplified wing-body configuration at an $\alpha$ of $4 \mathrm{deg}$. This configuration was specifically chosen because the vertical location of its $c p$ and center of gravity $(c g)$ are in close proximity, which results in a small moment arm for the pitching moment due to drag. This means that for this configuration, the drag force should have a significantly small contribution to the pitching moment and as a result, it can be calculated relatively accurately using only the axial lift distribution. Therefore, the calculated $c p_{x}$ based on $A_{\mathrm{e}}^{\text {lift }}$ should closely match the $c p_{x}$ calculated from the surface pressure distribution (which also accounts for the moment contribution due to drag).

A geometry deformation is performed by applying a wing tip twist of $-1 \mathrm{deg}$ and $+1 \mathrm{deg}$. No twist is applied at the wing root and the twist distribution is allowed to vary linearly across the span. The sensitivity comparison provided in Fig. 4 shows good agreement between the $c p_{x}$ calculated directly from the CFD surface pressure distribution, the $c p_{x}$ that was calculated from $A_{\mathrm{e}}^{\text {lift }}$, and the $c p_{x}$ that was calculated from surrogate $A_{\mathrm{e}}^{\text {lift }}$. The maximum difference between the $c p_{x}$ based on the $A_{\mathrm{e}}^{\text {lift }}$ and the actual $c p_{x}$ is 0.87 percent. This result confirms the assumption that if the contribution of drag to pitching moment is small then $A_{\mathrm{e}}^{\text {lift }}$ is sufficiently accurate to predict $c p_{x}$. In addition, Fig. 4 shows that the $c p_{x}$ which is calculated from surrogate $A_{\mathrm{e}}^{\text {lift }}$ (denoted by the black data line) also predicts the sensitivity in $c p_{x}$ accurately because the difference in $\Delta A_{\mathrm{e}}$ between the baseline and

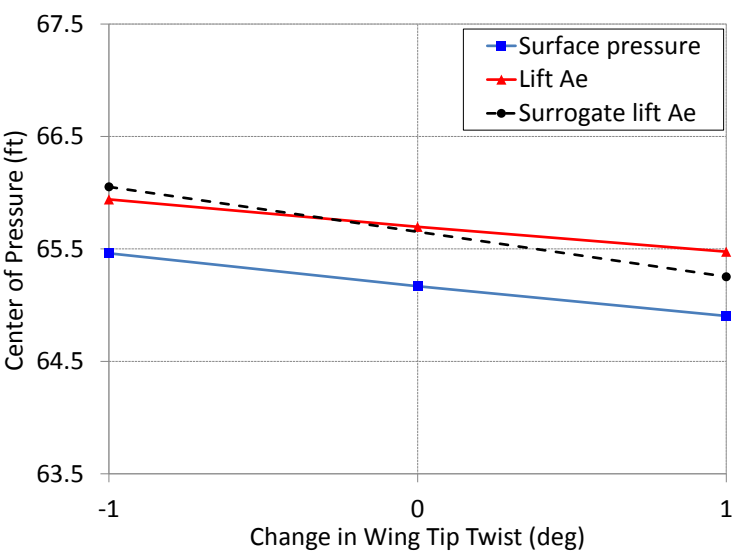

Figure 4. Verification of center of pressure sensitivity for simplified wing-body configuration. deformed configurations is small.

\section{Sensitivities Based on Shaping for a Demonstrator Concept}

A second study is used to verify the sensitivity of $c p_{x}$ for a low-boom demonstrator concept with respect to main wing camber, and horizontal tail twist variations. The low-boom demonstrator which uses a flow-through nacelle is shown in Fig. A-1 of the Appendix.

First, the main wing camber at the root midchord is varied incrementally by $0.5 \mathrm{ft}$ from $-1 \mathrm{ft}$ to $+1 \mathrm{ft}$. The horizontal tail tip twist is varied incrementally by $0.5 \mathrm{deg}$ from $-1 \mathrm{deg}$ to $+1 \mathrm{deg}$. The root chord of the horizontal tail is untwisted and the twist distribution is allowed to vary linearly across the span. The sensitivities for main wing camber and horizontal tail twist variations are provided in Figs. 5(a) and 5(b), respectively. Due to the large moment arm difference, the change in $c p_{x}$ resulting from wing camber variation is small relative to the change due horizontal tail twist. Nonetheless, in both cases the sensitivity of $c p_{x}$ that is calculated with the surrogate $A_{\mathrm{e}}^{\text {lift }}$ shows good agreement with the sensitivity of $c p_{x}$ calculated using the CFD-based surface pressure distribution.

\section{Practical Design of a Demonstrator Concept}

A third verification case was conducted for a practical design of a low-boom demonstrator concept. The concept used (shown in Fig. A-2 of the Appendix) is $130 \mathrm{ft}$ in length and 23,000 lbs at the start of cruise. This concept is an earlier design iteration of the concept shown in Fig. A-1 of the Appendix. As before, a flow-through nacelle is used for simplification. This concept was originally shaped to match the low-boom target $A_{\mathrm{e}}$ at a cruise altitude of $45,000 \mathrm{ft}$ as shown in Fig. 6(a). The $c g_{x}$ at the start of cruise was calculated to be $95 \mathrm{ft}$ using low-fidelity methods which assume an ideal fuel distribution, and $c p_{x}$ was calculated from surface pressure distribution to be $102 \mathrm{ft}$ (untrimmed). The surrogate $c p_{x}$ for the low-boom target at 45,000 ft was calculated to be $102.6 \mathrm{ft}$ and confirms that matching this target with lift tailoring does not produce a trimmed configuration.

A target optimization was conducted to obtain a new target that is trim-feasible with a cruise altitude at approximately 55,000 ft. The surrogate $c p_{x}$ for this new target was calculated to be $94.1 \mathrm{ft}$. This means that the baseline configuration is expected to be trimmed and low-boom if it is shaped through lift tailoring to match the new target. First, $\alpha$ was adjusted from $2.95 \mathrm{deg}$ to $3.70 \mathrm{deg}$ to meet the $C_{L}$ requirement at the new cruise altitude of 55,000 ft, resulting in the new baseline $A_{\mathrm{e}}^{\text {rev }}$ shown in Fig. 6(b). 


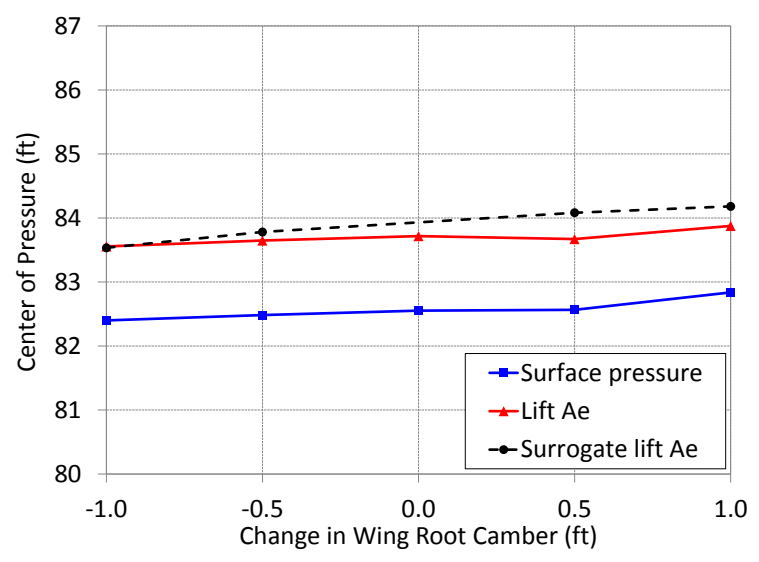

(a) Sensitivity to wing camber.

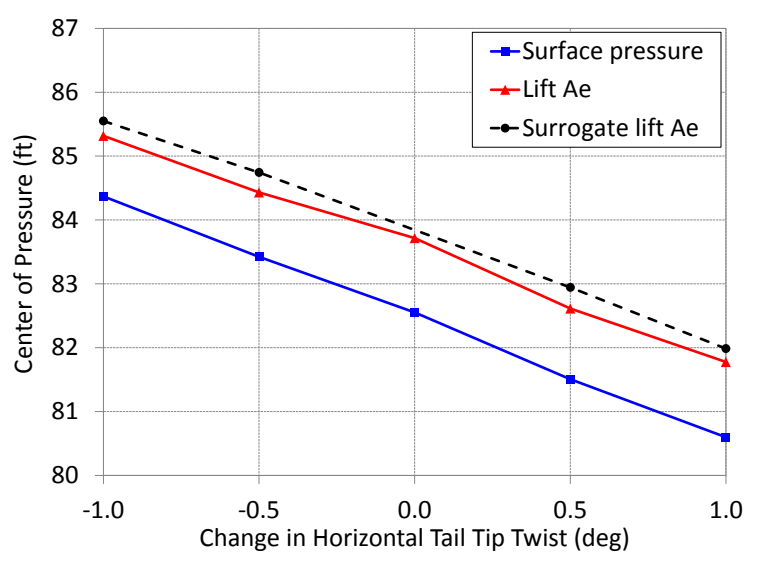

(b) Sensitivity to horizontal tail twist.

Figure 5. Verification of center of pressure sensitivity for a low-boom demonstrator concept.

The $c p_{x}$ for the baseline configuration at an altitude of $55,000 \mathrm{ft}$ before re-design was calculated to be $100.9 \mathrm{ft}$. For reference, the surrogate $c p_{x}$ of the baseline configuration at $55,000 \mathrm{ft}$ was calculated to be $101.1 \mathrm{ft}$. This match indicates that the surrogate $c p_{x}$ is sufficiently accurate despite the large variation in $\alpha$. The baseline configuration was then shaped using the mixed-fidelity design process through lift tailoring of the wing and horizontal tail to closely match the target $A_{\mathrm{e}}$ at $55,000 \mathrm{ft}$ as shown in Fig. 6(b). The re-design also consisted of adjustments in $\alpha$ which resulted in a final $\alpha$ of $3.55 \mathrm{deg}$. The newly re-designed concept was confirmed to be relatively close to trimmed with a $c p_{x}$ that was calculated to be $95.3 \mathrm{ft}$ (a shift in $c p_{x}$ of $5.6 \mathrm{ft}$ ) based on the surface pressure distribution. This example is presented only to demonstrate the capability of the trim-feasible target optimization process. Ideally, the designer would not shape a configuration to match a low-boom target which is not expected to produce a trimmed configuration before re-shaping the concept to match a trim-feasible target.

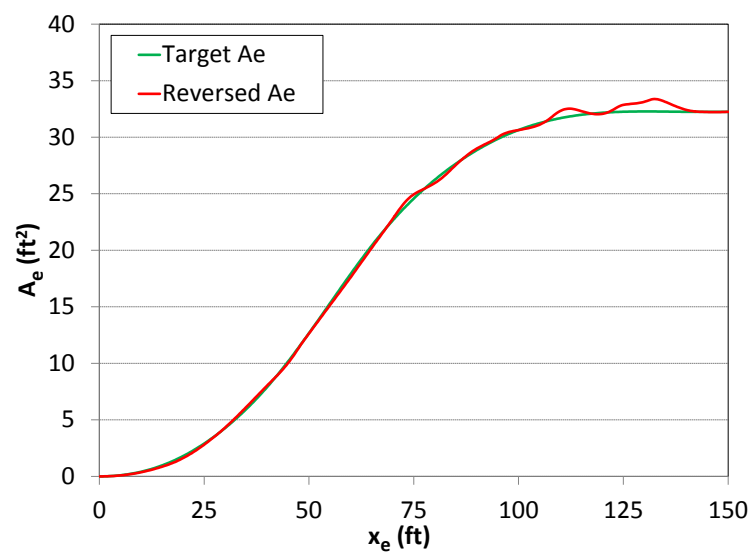

(a) Baseline configuration at a cruise altitude of 45,000 ft.

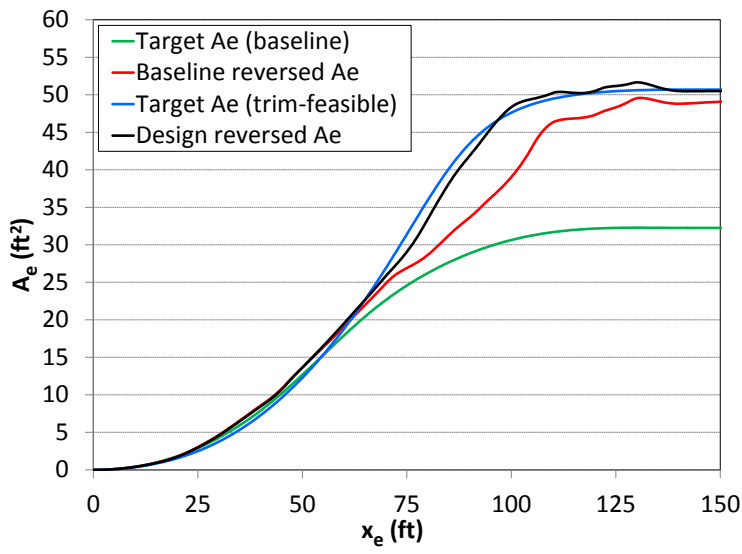

(b) Baseline and design configurations at a cruise altitude of $55,000 \mathrm{ft}$.

Figure 6. Analysis of equivalent area.

\section{Conceptual Design Process of a Low-Boom Demonstrator}

The design work described in this section represents an early conceptual design effort which is intended to define a baseline configuration that is trimmed during cruise and feasible for low-boom. The objective here is not to present the more detailed design work required to numerically match a low-boom target. Low-boom and trim feasibility is based on the determination that the design is sufficiently close to the target in the $A_{\mathrm{e}}$ design space. Feasibility is assessed by comparison of the deviation in $A_{\mathrm{e}}^{\text {rev }}$ of a configuration from the target $A_{\mathrm{e}}$, as well as the location and effectiveness of the aircraft components to provide sufficient design authority in regions with greater $A_{\mathrm{e}}$ deviation. The baseline configuration shown in Fig. A-1 of the Appendix has already been presented during the verification of the formulation 
(Section III). This baseline geometry is based on a shortened version of a trimmed $130 \mathrm{ft}$ concept similar to that shown in Fig. A-2 of the Appendix. Note that there are some differences in planform between the two configurations, and the inlet of the embedded engine has been shortened to reduce the complexity and efficiency losses.

The flight conditions at the start of cruise for the baseline configuration are specified as 21,000 lbs weight, Mach number of 1.6, and an altitude of 50,000 ft. However, the cruise altitude (and consequently the $\alpha$ ) is allowed to vary depending on the requirements set by the trim-feasible target optimization.

\section{A. Mass Properties, Propulsion System, and Trim Analysis}

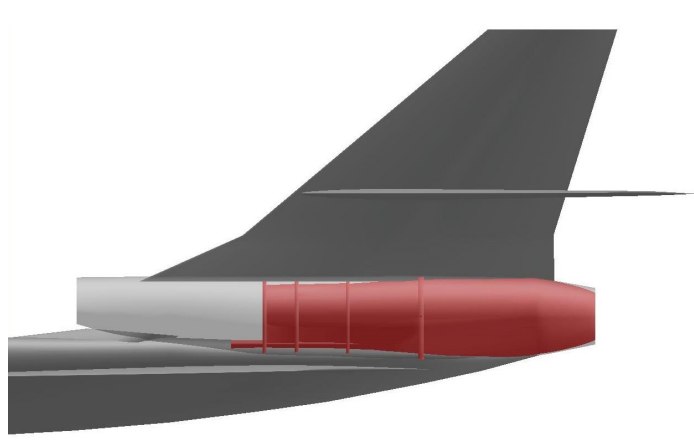

Figure 7. Propulsion system.

The conceptual design methods used to calculate mass properties, $c g$, and mission performance are described in Ref. 16. The propulsion system shown in Fig. 7 is an F404-402 engine weighing 2,272 lb. Engine performance is calculated with the Numerical Propulsion System Simulation ${ }^{17}$ (NPSS) computer program based on publicly available data. The landing main gear is located at $x=82.5 \mathrm{ft}$, which is the most aft location that is favorable for trim while also satisfying (i) the requirement to be located aft of the empty weight (unless ballast is used), and (ii) landing and takeoff static stability, and tail deflection requirements for rotation.

An initial trim analysis is performed for the baseline configuration to determine the forward shift in $c p_{x}$ required to achieve trim. Table 1 lists the $c g_{x}$ and $c p_{x}$ for the baseline configuration at the start of cruise. The $c p_{x}$ calculation in Table 1 is based on the Cart3D surface pressure distribution. The cruise most aft $c g_{x}$ is located fore of the $c p_{x}$ indicating that this concept is inherently unable to trim through weight re-distribution. This means that in order for the $c g_{x}$ to match the $c p_{x}$, the fuel would have to be located aft of the physical aircraft. The baseline configuration was found to be impossible to trim even with the use of a 40 percent fuel fraction in the horizontal and vertical tail.

\section{B. Description of Low-Boom Design and Trim Process}

The previous $c g_{x}$ calculation for the baseline configuration is used as a reference value for the required location of the surrogate $c p_{x}$ during the trim-feasible target optimization process. The baseline configuration and mission is assumed to have an initial cruise altitude of 50,000 ft based on previous trim-feasible target exploration with this size aircraft. At this flight condition, the baseline configuration has a cruise most aft $c g_{x}$ of $84.5 \mathrm{ft}$ and $c p_{x}$ of $86.1 \mathrm{ft}$, as shown in Table 1. The required shift in $c p_{x}$ is $1.6 \mathrm{ft}$. This is a relatively small shift due to more aggressive weight distribution toward the back of the aircraft when compared to the earlier $130 \mathrm{ft}$ concept. Also note that this most aft $c g_{x}$ is idealized, since there is no tank definition, and the fuel is assumed to always occupy the most aft regions of the fuselage.

The NSGA-II optimizer is used to minimize the perceived loudness level and surrogate $c p_{x}$ as equally weighted objectives. The change in surrogate $c p_{x}$ is calculated by subtracting the surrogate $c p_{x}$ of a candidate target $A_{\mathrm{e}}$ from the surrogate $c p_{x}$ of the baseline configuration. Recall that the surrogate $c p_{x}$ of the baseline configuration is calculated using the CFD-based $A_{\mathrm{e}}^{\text {lift }}$, which assumes that the drag contribution to the moment is small. A Pareto front is then generated by the NSGA-II optimizer as shown in Fig. 8. This plot is used to identify candidate $A_{\mathrm{e}}$ targets which are likely to satisfy the trim condition of the next design iteration. Here, the target $A_{\mathrm{e}}$ with the lowest PLdB that is capable of translating the $c p_{x}$ forward by the required distance is selected. In this case, the chosen target $A_{\mathrm{e}}$ which is shown in Fig. 9(a) (denoted by the red data marker) has a loud-

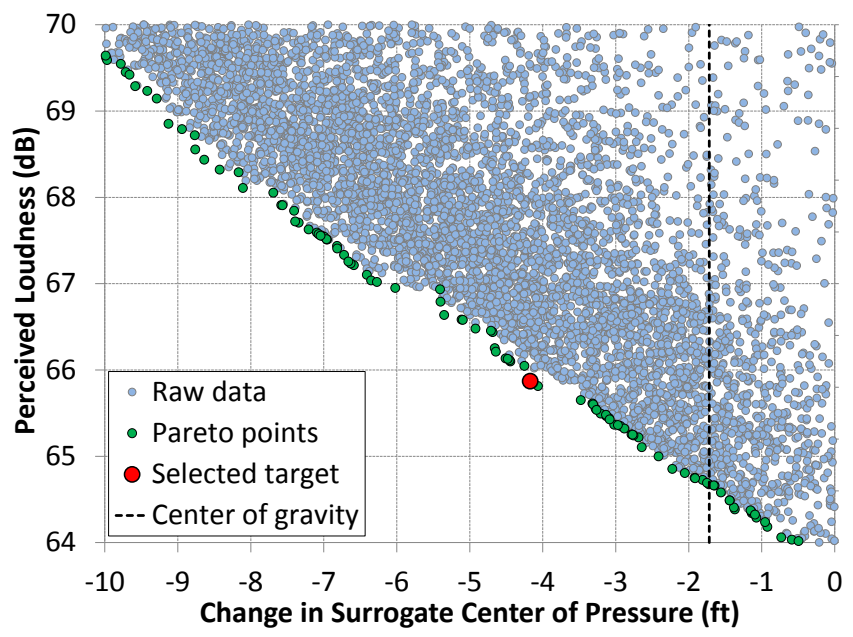

Figure 8. Pareto frontier of loudness and change in $c p_{x}$. 
ness of $65.9 \mathrm{PLdB}$, and is expected to translate the $c p_{x}$ forward by $4.2 \mathrm{ft}$. Note that the chosen target also produces a more aggressive shift in $c p_{x}$ than required to allow a margin for the uncertainty associated with the low-fidelity cruise most aft $c g_{x}$ calculation. The cruise altitude for the target $A_{\mathrm{e}}$ is calculated to be $51,707 \mathrm{ft}$ based on the end value of the surrogate $A_{\mathrm{e}}^{\text {lift }}$, and assuming that the cruise weight and Mach number remain unchanged.

The baseline configuration is now shaped with the mixed-fidelity $A_{\mathrm{e}}$ approach. A parameterization scheme based on camber deformation is placed on the main wing, with the camber design variable locations shown in Fig. A-4 of the Appendix. The camber is then optimized interactively by tailoring the lift distribution to match the trim-feasible target $A_{\mathrm{e}}$. A comparison of the wing camber deformation for the baseline and design configurations is provided in Fig. 10. The $A_{\mathrm{e}}^{\text {rev }}$ and ground signature $(\Delta p)$ for the design configuration (denoted by the red data lines) are provided in Figs. 9(a) and 9(b), respectively. The calculation of $c p_{x}$ based on the surface pressure distribution of the design configuration confirms a forward translation of approximately $4.2 \mathrm{ft}$ in $c p_{x}$. The $c p_{x}$ of the design provided in Table 1 is not expected to vary significantly when more detailed design shaping is performed to fully match the low-boom target because of the relatively close match in the $A_{\mathrm{e}}$ design space.

Table 1. Mass and aerodynamic properties of baseline and design configurations.

\begin{tabular}{lcccc}
\hline Configuration & $\begin{array}{c}\text { Altitude } \\
(\mathrm{ft})\end{array}$ & $\begin{array}{c}\text { Cruise } \\
\text { most aft } c g_{x}(\mathrm{ft})\end{array}$ & $\begin{array}{c}\text { Cruise } \\
c p_{x}(\mathrm{ft})\end{array}$ & $\begin{array}{c}\text { Static } \\
\text { Margin }\end{array}$ \\
\hline \hline Baseline & 50,000 & 84.5 & 86.1 & \\
Baseline & 51,707 & 84.5 & 86.1 & -1.72 \\
Design & 51,707 & 84.5 & 81.9 & -9.30 \\
\hline
\end{tabular}

More detailed modeling of fuel tank placement, and more accurate weight calculations later identified the cruise most aft $c g_{x}$ to be $82.0 \mathrm{ft}$ for the fuel tank configuration shown in Fig. A-3 of the Appendix. A total of 6 fuselage and 4 wing fuel tanks are installed with consideration of structural members. The fuel fraction and density of the fuel tanks is 0.8 and $50.5 \mathrm{lb} / \mathrm{ft}^{3}$, respectively. The more accurate $c g_{x}$ is located fore of the original low-fidelity cruise most aft $c g_{x}$ of $84.5 \mathrm{ft}$. This underscores the importance of allowing a surrogate $c p_{x}$ margin when selecting a low-boom target in the absence of more accurate weight calculations. The original $c p_{x}$ margin of $4.2 \mathrm{ft}$ for the chosen low-boom target proved to be sufficient to maintain a trimmed state at cruise. The static margin reported in Table 1 also shows that both baseline and final design configurations are stable.

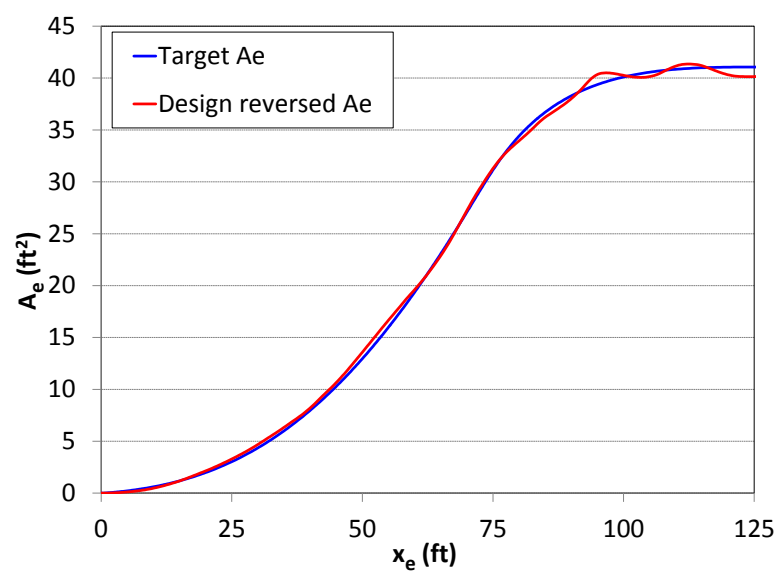

(a) Equivalent area.

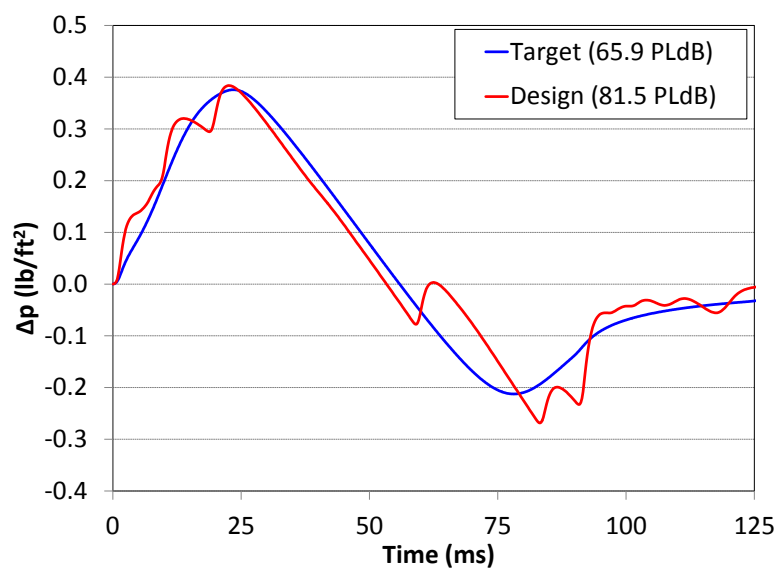

(b) Ground signature.

Figure 9. Results of concept re-design with mixed-fidelity design process.

\section{Concluding Remarks}

A new low-boom target generation approach that accounts for a trim requirement has been presented. The trim-feasible, low-boom target optimization uses a surrogate $A_{\mathrm{e}}^{\text {lift }}$ based on a target $A_{\mathrm{e}}$. This surrogate $A_{\mathrm{e}}^{\text {lift }}$ provides an approximation of $c p_{x}$ for a configuration with a $A_{\mathrm{e}}^{\text {rev }}$ that matches the target $A_{\mathrm{e}}$ through lift tailoring. The formulation assumes that (i) the configuration has a large fineness ratio which 


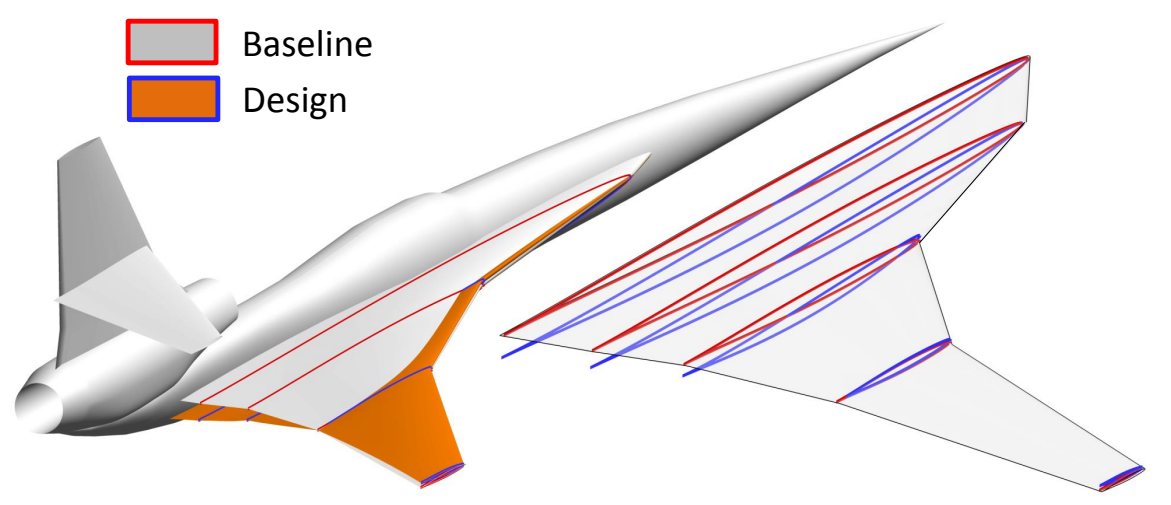

Figure 10. Comparison of baseline and design wing camber shape.

makes the lift force the dominant contributor to pitching moment, and (ii) the change in $\Delta A_{\mathrm{e}}$ between the baseline and design configurations is assumed to be small.

The sensitivity of surrogate $c p_{x}$ to shape changes has been verified with three case studies. The first case study verifies that the error between the surrogate $c p_{x}$ and the $c p_{x}$ that is based on surface pressure distribution is less than 1 percent for a configuration that satisfies all formulation assumptions. The second case study shows that the sensitivity of surrogate $c p_{x}$ to changes in lifting surfaces for a realistic low-boom demonstrator concept is also sufficiently accurate to the $c p_{x}$ calculated from the surface pressure distribution. Therefore, shaping of the configuration to match the low-boom target is also expected to generate the desired translation in $c p_{x}$. The third case study demonstrates that a non-trimmed but lowboom feasible concept can be re-designed using a trim-feasible, low-boom target to achieve trim while maintaining the desired low-boom characteristics.

Finally, the early conceptual design of a trimmed and low-boom feasible demonstrator concept has been presented. The new low-boom target optimization process was used to generate a target $A_{\mathrm{e}}$ that is capable of driving the low-boom design toward a trimmed state. This low-boom target $A_{\mathrm{e}}$ also defines the required start of cruise altitude. In addition, the generated Pareto front of the loudness level and surrogate $c p_{x}$ for a baseline configuration can quantify the likelihood of achieving a low-boom design that is capable of trim. Lift tailoring based on camber deformation of the main wing was used to approximately match the trim-feasible target $A_{\mathrm{e}}$. The re-design of the configuration to match the target $A_{\mathrm{e}}$ confirmed the expected forward shift in $c p_{x}$ of approximately $4.2 \mathrm{ft}$. The final design configuration was shown to be low-boom feasible, trimmed, and stable at cruise, and capable of meeting all requirements for the demonstrator mission definition.

\section{Appendix: Additional Figures}

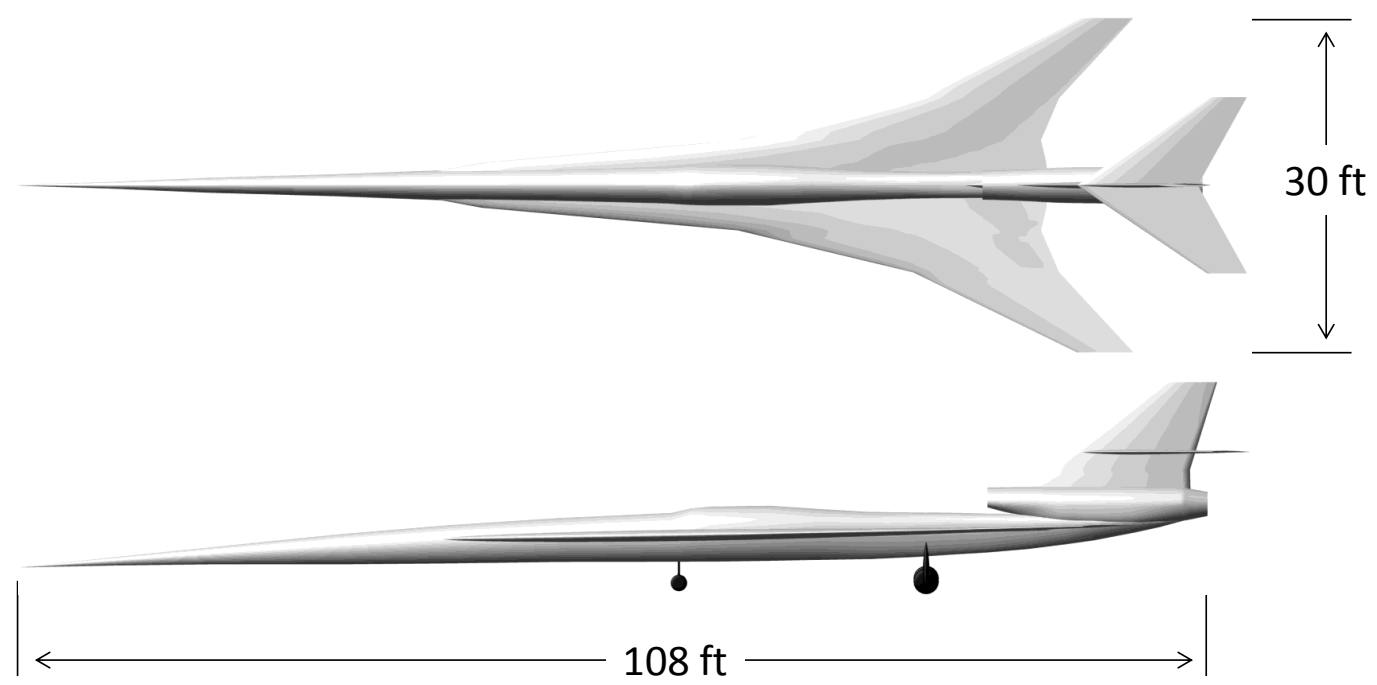

Figure A-1. Baseline low-boom demonstrator concept (108 ft). 


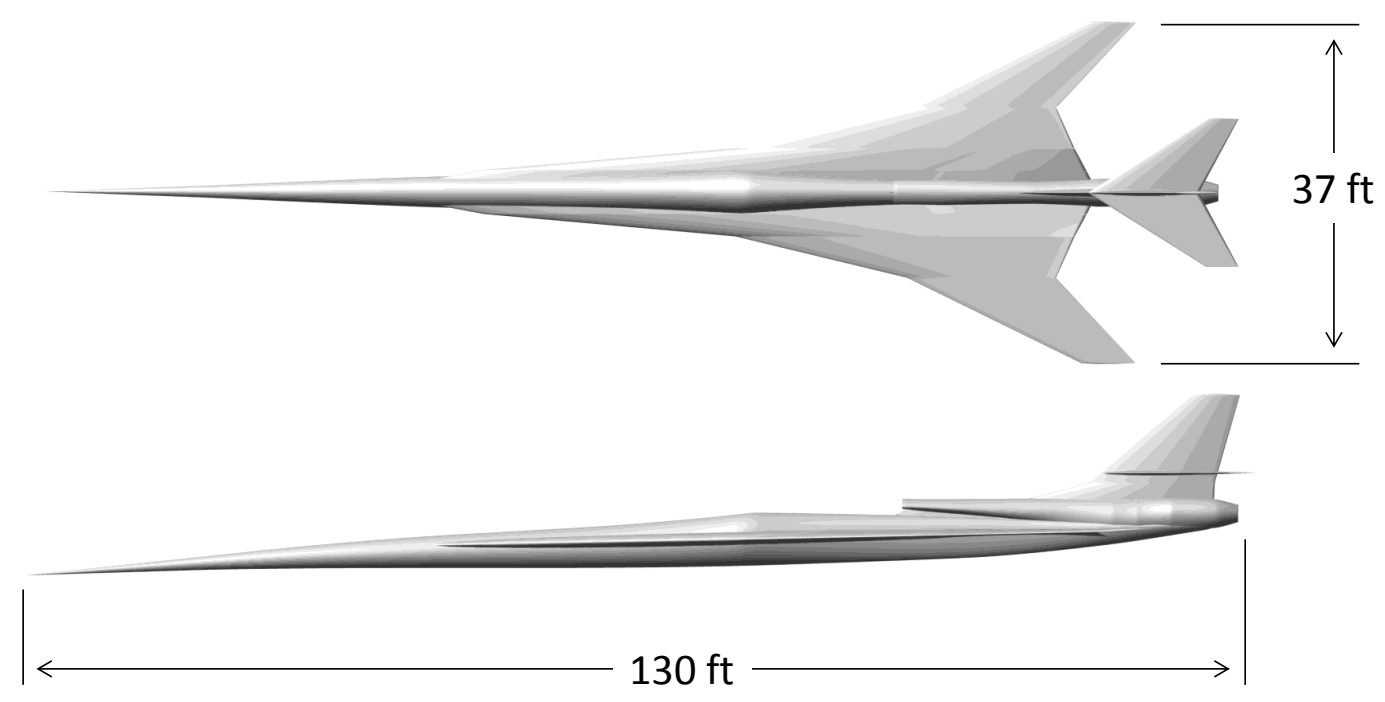

Figure A-2. Low-boom demonstrator concept (130 ft).

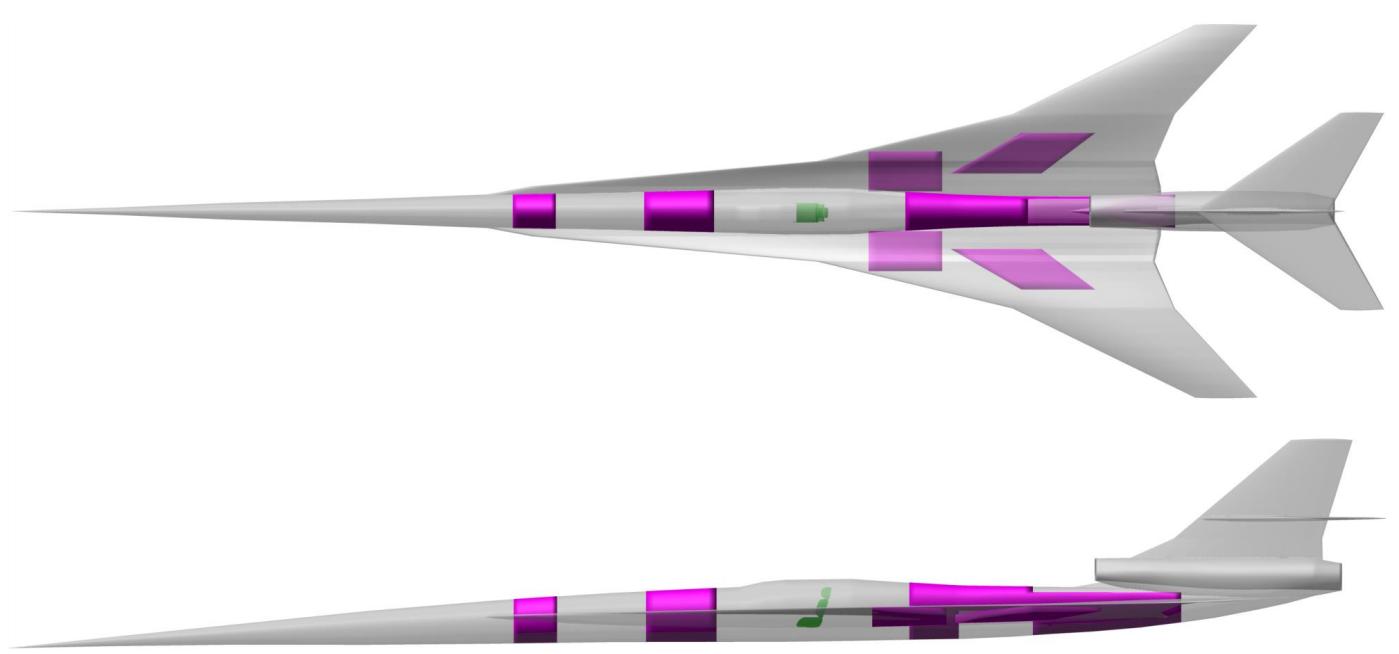

Figure A-3. Fuel tank placement for low-boom demonstrator concept (108 ft).

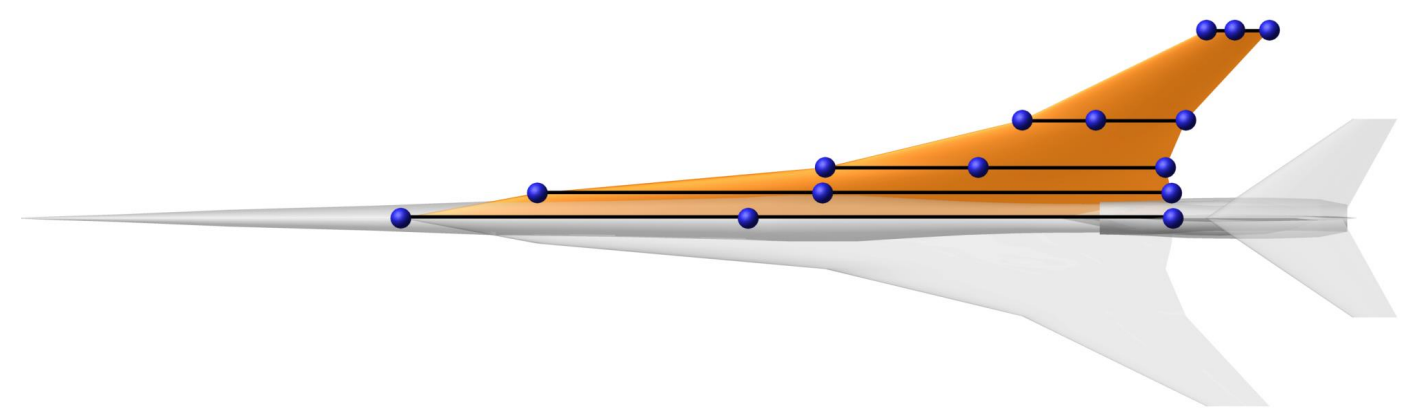

Figure A-4. Wing camber parameterization scheme.

\section{Acknowledgement}

The authors would like to thank Mathias Wintzer (Analytical Mechanics Associates) and Wu Li (NASA Langley Research Center) for their valuable input regarding formulation and optimization objectives used during the generation of trim-feasible targets. The authors would also like to thank $\mathrm{Wu}$ Li for his contribution to low-boom feasibility studies for the layout used in the presented low-boom demonstrator concept. 


\section{References}

${ }^{1}$ Wintzer, M., "Optimization and Adjoint-Based CFD for the Conceptual Design of Low Sonic Boom Aircraft," Ph.d. dissertation, Stanford University, August 2012

${ }^{2}$ Aftosmis, M. J., Nemec, M., and Cliff, S. E., "Adjoint-Based Low-boom Design with Cart3D," AIAA-2011-3500, June 2011.

${ }^{3}$ Rallabhandi, S. K., "Application of Adjoint Methodology to Supersonic Aircraft Design Using Reversed Equivalent Areas," AIAA-2013-2663, June 2013.

${ }^{4}$ Ordaz, I. and Li, W., "Integration of Off-Track Sonic Boom Analysis in Conceptual Design of Supersonic Aircraft," Journal of Aircraft, Vol. 51, No. 1, January-February 2014, pp. 23-28

${ }^{5} \mathrm{Li}$, W. and Shields, E., "Generation of Parametric Equivalent-Area Targets for Design of Low-Boom Supersonic Concepts," AIAA-2011-462, January 2011

${ }^{6}$ Ordaz, I. and Li, W., "Adaptive Aft Signature Shaping of a Low-Boom Supersonic Aircraft Using Off-Body Pressures," AIAA-2012-0020, January 2012.

${ }^{7} \mathrm{Li}, \mathrm{W}$. and Rallabhandi, S. K., "Inverse Design of Low-Boom Supersonic Concepts Using Reversed Equivalent-Area Targets," Vol. 51, 2014, pp. 29-36.

8 "ModelCenter and Optimization Tools," Phoenix Integration, http://www.phoenix-int.com/ [cited March 2014]

${ }^{9}$ Ordaz, I. and Li, W., "Using CFD Surface Solutions to Shape Sonic Boom Signatures Propagated from Off-Body Pressure," AIAA-2013-2660, June 2013.

${ }^{10}$ Aftosmis, M. J., "Cart3D Resource Website," http://people.nas.nasa.gov/ aftosmis/cart3d/cart3Dhome.html [cited October 2011].

${ }^{11}$ Aftosmis, M. J., Berger, M. J., and Adomavicius, G., "A Parallel Multilevel Method for Adaptively Refined Cartesian Grids with Embedded Boundaries," AIAA-2000-0808, January 2000.

12 "USM3D Users Manual, Version 6.0," http://tetruss.larc.nasa.gov/usm3d [cited March 2014].

${ }^{13}$ Rallabhandi, S. K., "Advanced Sonic Boom Prediction Using Augmented Burger's Equation," AIAA-2011-1278, January 2011.

${ }^{14}$ Walkden, F., "The Shock Pattern of a Wing-Body Combination Far from the Flight Path," Aeronautical Qtr, Vol. 9, No. 2, 1958.

${ }^{15}$ Srinivas, N. and Deb, K., "Muiltiobjective Optimization Using Nondominated Sorting in Genetic Algorithms," Evolutionary Computation, Vol. 2, No. 3, Fall 1995, pp. 221-248.

${ }^{16}$ Geiselhart, K. A., Ozoroski, L. P., Fenbert, J. W., Shields, E. W., and Li, W., "Integration of Multifidelity Multidisciplinary Computer Codes for Design and Analysis of Supersonic Aircraft," AIAA-2011-465, January 2011.

${ }^{17}$ Lytle, J., Follen, G., Naiman, C., Evans, A., Veres, J., Owen, K., and Lopez, I., "Numerical Propulsion System Simulation (NPSS) 1999 Industry Review," NASA TM-2000-209795, August 2000 